\title{
The Road towards Polarized Antiprotons
}

\author{
Hans Ströher ${ }^{1}$ \\ Institut für Kernphysik, Jülich Center for Hadron Physics, Forschungszentrum Jülich GmbH \\ 52425 Jülich, Germany \\ E-mail: h.stroeherefz-juelich.de
}

\section{Paolo Lenisa}

Universita di Ferrara and Istituto Nazionale di Fisica Nucleare

44100 Ferrara, Italy

E-mail: Ienisalfe.infn.it

\section{Frank Rathmann}

Institut für Kernphysik, Jülich Center for Hadron Physics, Forschungszentrum Jülich GmbH 52425 Jülich, Germany

E-mail: f.rathmannefz-juelich.de

The PAX-Collaboration is pursuing studies to find a method for producing an intense beam of polarized antiprotons. The following write-up gives a physics motivation, the possible methods and previous tests that have been performed. Currently spin-filtering in a suitable storage ring is the only viable method to achieve this goal. PAX has recently performed a spin-filtering measurement with protons in the COSY storage ring at Forschungszentrum Jülich (Germany) as a step towards an experiment with antiprotons, planned at CERN/AD. An outlook is also given as to what the possibility would be for exploiting a polarized antiproton beam in HESR at FAIR. All of the issues are discussed in a concise way - for more details the reader is referred to the given references.

8th International Conference on Nuclear Physics at Storage Rings (Stori11) Laboratori Nazionali di Frascati dell'INFN, Italy

October 9-14, 2011

1 Speaker; for the PAX-Collaboration 


\section{Introduction}

The demand for polarized antiprotons was put onto the agenda as soon as first beams of antiprotons became available almost 30 years ago. Although polarized antiprotons from the parity-violating decay of anti- $\Lambda^{0}$ hyperons were used in an exploratory Fermilab experiment in 1996, the intensity - about $5 \times 10^{5}$ particles per spill (20 s) [1] - is much too small to be useful in beam fixed-target experiments and even more for colliding beams. The lack of intense beams of polarized antiprotons was considered a shortcoming of existing facilities like, e.g., LEAR at CERN. Several methods to polarize antiprotons were considered in topical workshops held in Bodega Bay, USA (1985) [2], Daresbury, UK (2007) [3] and Bad Honnef, Germany (2008) [4]. In 1993, the method of spin-filtering was tested with protons by the FILTEX collaboration [5] and proposed to be used for antiprotons as well. With the planned FAIR project at GSI and the HESR storage ring for antiprotons, the PAX collaboration was formed in 2005 to take on the issue again, proposing a physics program with the HESR, upgraded to an asymmetric polarized proton polarized antiproton collider [6]. In the following years, PAX has been working to establish a method to produce an intense beam of polarized antiprotons - in what follows, a status report will be given.

\section{The physics case for polarized antiprotons}

\subsection{Transversity}

Transversity is the remaining frontier of $\mathrm{k}_{\mathrm{T}}$-independent nucleon structure functions. The "golden channel" to study transversity is a measurement of the double spin asymmetry A $A_{T T}$ in polarized proton polarized antiproton Drell-Yan (DY) production [7, 8]. These reactions are dominated by valence quark and antiquark annihilations in contrast to the proton-proton case (which might be used to extract sea-quark transversity, e.g., at RHIC). Being chiral-odd, transversity is not accessible in deeply-inelastic scattering (DIS). Unlike semi-inclusive DIS (SIDIS), transversely polarized DY does not involve fragmentation functions to extract the transversity structure function, thus the important but largely unknown piece of nucleon spin structure will be accessible in a unique way.

\subsection{Other parton distribution functions}

Drell-Yan production will not only help to determine transversity, but it can, e.g., also probe the leading-twist transverse momentum-dependent parton distribution function $\mathrm{h}_{1 \mathrm{~T}}$ perp [9] or the higher-twist so called Wandzura-Wilczek contribution via the double-spin asymmetry $\mathrm{A}_{\mathrm{LT}}[10]$.

\subsection{Nucleon time-like form factors}

Form factors are important observables to investigate the structure of the nucleon. The use of polarization in electron scattering measurements of the ratio of space-like magnetic and electric proton form factors has recently resulted in an unexpected $\mathrm{Q}^{2}$-dependence, which also makes experiments in the time-like region (realized in annihilation reactions p p_bar $\longleftrightarrow \rightarrow \mathrm{e}^{+} \mathrm{e}^{-}$) increasingly interesting. In double polarized measurements not only the moduli of electric and magnetic time-like form factors but also their relative phase will be accessible [11]. 


\section{Possibilities to polarize antiprotons and previous tests}

\subsection{Polarization methods}

The polarization methods can be divided into two broad classes: (i) selective flip and (ii) selective loss [12]. Both methods provide a polarized beam from an originally unpolarized one, but (i) has the advantage of essentially no loss of intensity, while (ii) explicitly relies on the different loss rates for the individual polarization states.

As mentioned above, there has been a series of topical meetings on the issue of finding a method to produce polarized antiprotons (see references given in the introduction). In recent years, a paper claiming a very high cross section for the polarization transfer from polarized electrons (positrons) to protons (antiprotons) at small relative energy has received a lot of attention [13] (see however [14, 15]. Since it is an elaborate task to perform a test with antiprotons and polarized positrons, we essentially turned the reaction around and made a depolarization measurement with polarized protons, interacting with unpolarized electrons (see sect. (3.2.) below).

More recently, B. Schoch has suggested to exploit the different spin-dependent pion photoproduction cross sections on the proton to either produce polarized anti-protons (for $\pi^{0}$ ) or antineutrons (for $\pi^{+}$) [16]. Since this method is based on measured cross sections, it should work, the question is, however, its technical feasibility.

\subsection{Test of spin-flip at COSY}

Since spin-flip would be particularly appealing as a method to polarize antiprotons, the PAX collaboration has put a lot of emphasis in scrutinizing (i.e. verifying or falsifying) the proposal in a dedicated COSY experiment by investigating the depolarization of an originally polarized beam of protons, which interacted with unpolarized electrons from the electron cooler at very low relative momenta. In short, the method can unfortunately not be used to produce polarized antiprotons - the cross sections are orders-of-magnitude smaller than was theoretically predicted by one group and in fact way-too-small [17] to provide significant spin-flip.

\subsection{Spin filtering}

Spin filtering is known to work for protons since the FILTEX measurement, performed at the TSR of MPI Heidelberg in the 1990's [5], which was later on proposed to be used for antiprotons in 2005 [18]. The PAX collaboration has taken on this proposal in connection with the FAIR/HESR project [19]. PAX has received broad support by the hadron physics community for its stepwise plan to (i) perform a test measurement with protons at COSY, repeating FILTEX for a beam energy around $50 \mathrm{MeV}$ and commissioning all equipment [20], and (ii) to do a first-time-ever spin filtering measurement with antiprotons at CERN/AD [21].

\section{Spin filtering}

\subsection{Set-up for spin filtering at COSY}

Spin filtering requires a beam of particles stored in a ring, which repeatedly interacts with a polarized hydrogen gas. In order to reach high target densities, the beam must traverse a storage cell, which in turn requires a low- $\beta$ section in the storage ring. Such a section has been set up at COSY and has commissioned in 2010.

During an eight week beamtime at COSY in Summer 2011, the PAX collaboration, in cooperation with the COSY accelerator group, has set up and optimized the cooler synchrotron 
and performed a spin filtering measurement. Details about the procedure can be found in the $\mathrm{PhD}$ Thesis of Ch. Weidemann [22] as well as the contribution to these proceedings by $\mathrm{D}$. Oellers [23].

\subsection{Antiproton spin filtering at CERN/AD}

The crucial step will be to perform a spin-filtering measurement with antiprotons. The only place to perform such an internal experiment in a storage ring will be the antiproton decelerator (AD) at CERN. The PAX collaboration has submitted a proposal to the corresponding CERN PAC (SPSC) [21] and is waiting for being approved. PAX has proposed a stepwise approach to install and test the necessary hardware into the $\mathrm{AD}$, but this will not be possible before the long CERN shutdown in 2013/14. The low- $\beta$ section for the AD is currently designed and built at Forschungszentrum Jülich.

\section{Further steps}

\subsection{Antiproton polarizer ring}

Once spin-filtering has been demonstrated to work for antiprotons and the optimal energy and additional parameters have been determined, the next task will be to design and build a dedicated antiproton polarizer ring (APR), and then to test it, presumably with protons. The APR must have a large acceptance and must be equipped with a low- $\beta$ section, an electron cooler and - supposedly - a Siberian snake for longitudinal filtering.

\subsection{Polarized-proton polarized-antiproton collider}

Finally, once the APR is available and has been tested with antiprotons, it could be part of an upgrade program at FAIR to convert the fixed-target HESR/PANDA into a(n) (a)symmetric proton-antiproton collider $[19,24]$ with both beams being polarized.

\section{Summary}

The PAX collaboration has started a program to find a method for providing an intense beam of polarized antiprotons. Some time ago, PAX has shown that spin-flip cannot be used. Pursuing spin-filtering, PAX has recently made significant progress with a test measurement with protons, which has been performed at COSY-Jülich. The next step, i.e. a spin-filtering measurement with anti-protons at CERN/AD is being prepared, but waiting for final approval of CERN.

The authors would like to acknowledge the excellent cooperation of the COSY accelerator crew in performing the challenging measurements at Jülich as well as the support of the Central Department of Technology (ZAT) of FZJ in designing and building hardware for the COSY (and CERN/AD) experiments. The PAX project is supported by JCHP-FFE funds of the Forschungszentrum Jülich, by EU I3HP (Hadron Physics - Polarized Antiprotons), and by an ERC Advanced Grant (H.S.). 


\section{References}

[1] A. Bravar et al., Single-Spin Asymmetries in Inclusive Charged Pion Production by Transversely Polarized Antiprotons, Physical Review Letters 77, 13 (1996).

[2] A.D. Krisch et al. (Eds.), Polarized Antiprotons, AIP Conference Proceedings 145 (1986).

[3] S. Chattopadhyay, D.P. Barber, N. Buttomore, G. Court, E. Steffens (Eds.), Polarized Antiproton Beams - How, AIP Conference Proceedings, Vol. 108, 2008, ISBN: 978-0-7354-0527-1.

[4] 409. WE-Heraeus Seminar, Polarized Antiprotons, Bad Honnef, Germany (2008), unpublished; see: http://www.fe.infn.it/heraeus/.

[5] F. Rathmann et al., New Method to Polarize Protons in a Storage Ring and Implictions to Polarized Antiprotons, Physical Review Letters 71, 9 (1993).

[6] PAX-Collaboration, founded in 2003, spokespersons P. Lenisa and F. Rathmann; see: http://www2.fz-juelich.de/ikp/pax/portal/

[7] A. Bianconi, Marco Radici, Monte Carlo Simulation of events with Drell-Yan lepton pairs from antiproton-proton collisions: the fully polarized case;

see: arXiv:hep-ph/0504261v1.

[8] Daniel Boer, Transversity Asymmetries;

see: arXiv:0808.2886vi.

[9] Jiacai Zhu, Bo-Qiang Ma, Probing the leading-twist transverse-momentum-dependent parton distribution function $h_{I T}$ perp via the polarized proton-antiproton Drell-Yan process, Physical Review D 82, 114022 (2010).

[10] Yuji Koike, Kazuhiro Tanaka, Shinsuke Yoshida, Drell-Yan double-spin asymmetry $A_{L T}$ in polarized pp_bar collisions: Wandzura-Wilczek contribution, Physics Letters B 668, 285 (2008).

[11] N.H. Buttimore, Jennings, Polarization Observables in leptin-antilepton-to-proton-antiproton reactions including lepton mass, European Physical Journal A 31, 9 (2007):

[12] E. Steffens, Polarized Antiprotons - The Quest for a Missing Tool, Spin Physics, AIP Conference Proceedings 1149, 80 (2009).

[13] Th. Walcher et al., A surprising method for polarising antiprotons, European Physical Journal A 34, 447 (2007).

[14] A.I. Milstein, S.G. Salinov, V.M. Strakhovenko, Polarization effects innon-relativistic ep scattering, Nuclear Instruments and Methods in Physics Research B 266, 3453 (2008).

[15] Th. Walcher et al., Erratum A surprising method for polarizing antiprotons, European Physical Journal A 39, 137 (2009).

[16] B. Schoch, A method to polarise antiprotons in storage ringsand create polarised antineutrons, European Physical Journal A 43, 5 (2010).

[17] D. Ollers et al., Polarizing a stored proton beam by spin flip?, Physics Letters B 674, 269 (2009).

[18] F. Rathmann et al., A Method to Polarized Stored Antiprotons to a High Degree, Physical Review Letters 94, 014801 (2005). 
[19] PAX-Collaboration, Antiproton-Proton Scattering Experiments with Polarization, Technical Proposal, Jülich (2005);

see: arXiv:hep-ex/0505054vi.

[20] PAX-Collaboration, Spin-Filtering Studies at COSY, Proposal \#192 to the COSY PAC (2009), Spokespersons D. Oellers and Chr. Weidemann;

see: http://www2.fz-juelich.de/ikp/pax/.

[21] PAX-Collaboration, Measurement of the Spin-dependence of the pp_bar Interaction at the ADRing, Proposal to the AD-PAC (SPSC) (2005), Spokespersons P. Lenisa and F. Rathmann; see: http://www2.fz-juelich.de/ikp/pax/.

[22] Ch. Weidemann, Preparations for the Spin-Filtering Experiments at COSY/Jülich, PhD Thesis, University of Cologne, Germany (2011).

[23] D. Oellers, Spin Filtering Experiment at COSY - First results, contribution to this conference (2011).

[24] Assia-Collaboration, A Study of Spin-dependent Interactions with Antiprotons: The Structure of the Nucleon, Proposal (2005)

see: arXiv:hep-ex/0507077v1. 